\title{
Hierarquia Urbana e Regionalização: uma proposta metodológica de ordenamento territorial para o Brasil
}

Ricardo Alexandrino Garcia ${ }^{1}$ Rodrigo Ferreira Simões ${ }^{2}$

Resumo: este artigo analisa a importância de uma nova proposta de regionalização para o Brasil, que deve considerar as especificidades locais, a paisagem cultural e de seus recursos, a complexidade crescente do espaço social e sua flexibilidade na organização e coordenação de território. O objetivo deste artigo é elaborar uma proposta de regionalização com base em centros urbanos e suas áreas de influência em vista da caracterização da Rede de Cidades e Seleção de nova centralidade no Brasil. Esta proposta visa identificar novos centros que possuem características econômicas que lhes permitam uma classificação mais elevada na hierarquia urbana brasileira, a partir de dois insumos básicos: Módulo III do Estudo Territorial Dimensão do Planejamento - o Ministério do Planejamento, Orçamento e Gestão - e as regiões de Influência dos Municípios definidos para 2007 - IBGE. O objetivo específico relacionado com a nova territorialidade econômica brasileira é o de identificar os atuais polos econômicos e potenciais e desejáveis centralidades urbanas, com vistas ao desenvolvimento. Esta divisão regional visa subsidiar o planejamento territorial brasileiro, apoiando a escolha e localização de investimentos direcionados para fortalecer centros emergentes e existentes capazes para criar nova centralidade para um Brasil policêntrico. Indicadores sintéticos são apresentados, juntamente os resultados preliminares que validam essa proposta metodológica, fornecendo uma visão geral da rede urbana brasileira.

Palavras-chave: hierarquia urbana; ordenamento territorial; regionalização; desenvolvimento; Brasil.

\footnotetext{
${ }^{1}$ Professor adjunto do Departamento de Geografia do Instituto de Geociências da UFMG

${ }^{2}$ Professor Associado do Departamento de Economia - Cedeplar/FACE/UFMG
} 


\section{1 - INTRODUÇÃO E OBJETIVOS}

O desenvolvimento socioeconômico exige envolvimento e legitimação de ações disruptivas e, portanto envolve tensão, eleição de alternativas e construção de trajetórias históricas, com horizontes temporais de curto, médio e longo prazo. Estudá-lo, portanto, exige ênfase em processos, estruturas e dinâmicas e a identificação dos agentes cruciais e das interações entre decisões e aquelas estruturas. É fundamental que esse processo transformador seja promovido simultaneamente em várias dimensões (produtiva, social, tecnológica, etc.) e em várias escalas espaciais, sejam elas local, regional, nacional ou global (DINIZ, 2006).

A complexidade subjacente ao espaço social - e a flexibilidade na sua organização e articulação - dificulta definições regionais rígidas e exige a combinação de critérios variados que dialoguem com as múltiplas espacialidades e territorialidades implícitas e/ou explícitas nas políticas setoriais e nos recortes territoriais. Reafirmando, as complexidades de análise territorial são enormes. Os instrumentos de intervenção sobre uma realidade localizada territorialmente podem estar em outra escala espacial, arena política, nível de governo, instância de poder. A escala deve ser vista como um recorte para a apreensão das determinações e condicionantes dos fenômenos sociais (LEMOS, 1991).

Neste sentido, é necessário construir, mesmo que introdutoriamente, estratégias multiescalares, pois cada problema pode ter suas diversas escalas espaciais específicas. Uma questão relevante da regionalização é definir qual a direção dos fluxos a ser privilegiada no esforço de planejamento. Do ponto de vista da integração econômica do território e do comércio exterior os fluxos inter-regionais são os mais relevantes, com ênfase nos eixos de integração física, especialmente o sistema de transportes. Do ponto de vista da integração urbano-regional, os fluxos intrarregionais devem ser enfatizados à medida que privilegiam as infraestruturas intermediárias que induzem o processo de integração urbano-regional e reforçam o papel polarizador do centro econômico dominante (CUNHA, SIMÕES \& PAULA, 2008).

As metodologias de regionalização mais recentes têm como grande mérito a ideia de pensar a ordenação territorial a partir da função de centralidade do urbano, o que permite entender a dinâmica territorial com base nos sistemas sub-regionais e as redes urbanas que os integram espacialmente (GARCIA, 2002). Essas novas centralidades deveriam ser selecionadas em função das forças do mercado, identificadas pelo potencial da expansão 
produtiva (agrícola, industrial, mineral, de serviços) e da intencionalidade política em termos de ordenamento do território, redução das desigualdades regionais, preservação ambiental, interesses de geopolítica e de soberania. Para o fortalecimento das novas centralidades desejadas, dois elementos se destacam: o sistema de transportes intrarregional e a concentração de equipamentos urbanos (DINIZ, 2000).

Combinando critérios de homogeneidade e heterogeneidade - de modo a conformar regiões-programa - é necessário estabelecer uma noção multiescalar e multicritério na concepção regional da ação de planejamento. A primeira - baseada na homogeneidade permite ver o Brasil segundo suas grandes diferenças macroespaciais, separando-se as áreas mais desenvolvidas das menos desenvolvidas, ou seja, áreas que constituem o centro e áreas que constituem a periferia. Permite também separar as áreas em expansão pelo seu dinamismo e potencialidade (Lemos 1991). O segundo critério teórico de recorte macroespacial do território, fundamentada na literatura consagrada a nível mundial, Lemos et al. (2003) parte da constatação de que as cidades, seus equipamentos e a rede de infraestrutura, especialmente transporte, estabelecem as forças polarizadoras, articulando e comandando o território.

Essa capacidade de comando, evidentemente, vai depender da escala (tamanho) e da hierarquia das cidades, da natureza de sua base produtiva, de sua localização e da infraestrutura de acessibilidade. $\mathrm{O}$ recorte em macrorregiões polarizadas combina a força polarizadora das grandes metrópoles, que constituem as atuais grandes centralidades do país, com suas áreas complementares (DINIZ, 1993). Tal regionalização permite visualizar e entender a atual estrutura macrorregional do país por meio da capacidade de comando do urbano sobre os grandes espaços subnacionais. Ela serve, também, para uma avaliação das distorções no ordenamento do território e indicar as alternativas para o fortalecimento de novas centralidades e das respectivas redes de infraestrutura e de equipamento urbano. Estas viriam permitir a mudança no ordenamento do território, a redução das desigualdades regionais, o aproveitamento das potencialidades regionais e $\mathrm{O}$ estabelecimento de políticas que permitam promover a integração macroespacial do território brasileiro e deste com a América do Sul. Ela deve ser vista como uma etapa necessária para a redução do peso de algumas megametrópoles e caminhar no sentido do fortalecimento de um sistema urbano policêntrico (GARCIA \& LEMOS, 2009). 
É, pois, do contraste entre o recorte da homogeneidade e da polarização que devem derivar os critérios de intervenção no território. Os mecanismos de polarização, especialmente a rede urbana e a infraestrutura e seus complementos são exatamente os instrumentos de intervenção para a redução das desigualdades identificadas pelos critérios de homogeneidade/diferença (MAGNACO, 1995; PACHECO, 1996). Essas são, pois, as razões pelas quais o recorte do território como fundamento para o planejamento deve partir dos dois fundamentos teóricos e metodológicos distintos para se atingir o objetivo único que é o uso das forças polarizadoras como instrumentos para a redução de desigualdades, identificadas pelos critérios de homogeneidade natural, econômica e social. Esses dois recortes devem servir de referência para as políticas macroespaciais, estruturadoras do território e voltadas para o seu ordenamento, guiado pelo objetivo ideal do policentrismo dos macropolos ou das grandes cidades.

O desenvolvimento policêntrico implica a adequada articulação e coordenação de ações políticas territoriais, setoriais e temporais. Política territorial é fundamental, já que a eleição de pólos de desenvolvimento que sejam capazes de dar características policêntricas à rede urbana brasileira o Brasil depende de um conhecimento sub-regional da malha urbana e da região de influências das cidades. A política setorial une o nacional ao sub-regional, ao promover uma política deliberada de seletividade setorial - que combinada à seletividade territorial acima mencionada - permita elencar conjuntos de investimentos tanto em nível nacional (investimentos em infraestrutura física) quanto em nível local (investimentos em funções e serviços urbanos). Neste contexto, a política temporal, por sua vez, aloca agrupamentos de investimentos no tempo. A seleção de novas centralidades vai subsidiar a política territorial que as elegerão como estratégicas para a construção de um Brasil mais balanceado (BAUDELLE, 2005).

A escala sub-regional, por outro lado, é a que mais se adéqua a construção de tipologias para efeito de políticas públicas e desenvolvimento regional, pois quebra a contiguidade regional que tradicionalmente é utilizada em macrorregiões com grandes diferenças internas. Ela permite compatibilizar as políticas de desenvolvimento das regiões menos desenvolvidas com a Política Nacional de Desenvolvimento Regional e a adequação dos recursos orçamentários ou fiscais para a política nacional operada em escala sub-regional. Ela permite, também, identificar e eleger um conjunto de novas subcentralidades que 
permitam modificar a estrutura da rede urbana, alterando o ordenamento do território com vistas a um Brasil policêntrico.

A rede de cidades é a estrutura que organiza o território e é o substrato que o condiciona, sobre o qual atuam as políticas públicas e os agentes sociais e econômicos que compõem a sociedade (REGIC, 2008). Está, portanto, diretamente relacionado à questão da territorialidade do desenvolvimento regional, buscando identificar os polos e centralidades atuais, potenciais e desejáveis, com vistas ao desenvolvimento. Uma melhor integração e um melhor ordenamento do território ocorrem em função das seguintes características: distribuição dos polos com seus equipamentos, serviços e atributos urbanos; potencial produtivo regional; infraestrutura; e capacidade, abrangência e força desses pólos em termos de polarização, comando e organização do território (Magnaco 1995).

O objetivo principal deste artigo foi, portanto, realizar uma caracterização da rede de cidades e seleção das novas centralidades, ou seja, identificar os polos e centralidades atuais, potenciais e desejáveis, com vistas ao desenvolvimento, baseados na regionalização por polos econômicos e suas áreas de Influência. Para tanto, buscou-se uma regionalização para o planejamento territorial brasileiro que permitisse subsidiar a escolha e localização de investimentos direcionados para o fortalecimento de polos existentes e novos polos que criassem novas centralidades para um Brasil policêntrico. Assim, os fins da regionalização estriam articulados às políticas públicas de planejamento regional no Brasil. O trabalho utilizou dois insumos básicos para a regionalização: i) o Módulo III do Estudo da Dimensão Territorial do MPOG (a regionalização que identificou 118 polos de desenvolvimento no Brasil); e ii) a Rede de Influência das Cidades de 2007 do IBGE (REGIC, 2008), que mapeou todas as áreas de influência dos centros urbanos brasileiros por meio de um da mensuração da intensidade de suas ligações (fluxos de bens e serviços)

A partir desta regionalização pré-definida, foi estabelecido um conjunto de indicadores relevantes que justifiquem a escolha dos novos polos reguladores. Tais indicadores são de: massa (seja do ponto de vista populacional, seja do ponto de vista da produção e da criação de riqueza), de conectividade (elos de relações ágeis com outros centros), de competitividade (fatores relevantes que capacitam diferenciadamente os territórios), de funções e instrumentos urbanos, entre outros, para a caracterização da rede de cidades. 
Os principais critérios para a escolha de novas centralidades são: 1) localização das cidades no contexto do território nacional e sul-americano, sendo este critério eliminatório; vale dizer, devem ser respeitadas as prioridades territoriais estabelecidas pela regionalização estratégica contida no MIII do estudo do MPOG; 2) distância das cidades de cada área de influência em relação aos seus macro e mesopólos; 3) densidade dos fluxos de bens e serviços destas áreas com os seus macro e mesopólos; 4) densidade populacional e econômica das cidades dentro de cada área de influência, definido a densidade econômica por um índice de terciarização e um índice de industrialização; 5) dinamismo econômico das cidades em cada área de influência; 6) simulação da configurações da rede de cidades estimada, baseada em projeções populacionais a partir das dinâmicas demográfica e econômica induzidas.

\section{2 - METODOLOGIA}

A metodologia escolhida para identificar potenciais centralidades urbanas em busca de um Brasil policêntrico procura estabelecer, a partir da regionalização contida no Módulo III do Estudo da Dimensão Territorial do Planejamento, do Ministério do Planejamento, Orçamento e Gestão, diferenciais entre os níveis hierárquicos da rede urbana brasileira, identificados no estudo sobre a Rede de Influência das Cidades de 2007 do IBGE, e uma possível reclassificação a partir de indicadores de potencial econômico/demográfico de mercado.

A proposta procura, por meio de um método de análise multivariado, identificar centralidades que possuiriam atributos, características econômicas que lhes permitissem classificação superior na hierarquia urbana brasileira. O pressuposto é que centralidades tipologizadas em um nível e que possuam elementos que poderiam coloca-la em outro nível hierarquicamente acima poderiam se configurar numa escolha eficiente do ponto de vista da inversão de recursos. Em outro registro, e complementarmente, as centralidades historicamente consolidadas e que possuíssem atributos potenciais que lhe dariam características de níveis inferiores no sistema urbano poderiam, também, ser objeto de atenção a fim de que não se sucateiem ativos relacionais importantes na configuração territorial brasileira.

O que aqui se apresentará a seguir é o conjunto de indicadores de potencial econômico/demográfico e a estrutura do modelo multivariado de Análise Discriminante. Os resultados preliminares foram apenas no sentido de validar esta proposta metodológica e ter uma visão panorâmica da rede urbana brasileira. Propositivamente, a incorporação de variáveis prospectivas por meio de simulações de cenários econômicos futuros por meio de um modelo econômico de Equilíbrio Geral Computável (EGC), bem como de projeções populacionais, no 
universo temporal de 2020, permitiu um diagnóstico prospectivo da configuração do sistema urbano brasileiro no horizonte de tempo delimitado, por meio da análise discriminante.

\section{Produto interno bruto per capita municipal (PIBPC)}

Esse indicador foi obtido pela razão entre o Produto interno bruto municipal de 2007 pela população estimada/recenseada no mesmo anos, ambas informações provenientes do IBGE:

$$
\mathrm{PIBPC}_{\mathrm{m}}=\frac{\mathrm{PIB}_{\mathrm{m}}}{\mathrm{POP}_{\mathrm{m}}}
$$

em que: PIBPC $_{\mathrm{m}}$ é o Produto interno bruto per capita municipal; $\mathrm{PIB}_{\mathrm{m}}$, o produto interno bruto municipal e $\mathrm{POP}_{\mathrm{m}}$ é a população do município

Taxa de crescimento anual do produto interno bruto municipal (TPIB)

Esse indicador foi obtido pelo cálculo da taxa de crescimento anual do PIB municipal entre 2002 e $2007:$

$$
\operatorname{TPIB}_{m, i f}=\left(\frac{P I B_{m, f}}{P I B_{m, i}}\right)^{1 / n}-1
$$

em que: $T_{P I B_{m, i f}}$ é a taxa anual de crescimento do Produto interno bruto entre 2002 e 2007; $P I B_{m, \beta}$ o produto interno bruto municipal de 2007; $P I B_{m, i}$, o produto interno bruto municipal de 2002 e $n$, o intervalo em anos.

\section{Taxa de crescimento anual do produto interno bruto per capita municipal (TPIBPC)}

Esse indicador foi obtido pelo cálculo da taxa de crescimento anual do PIBPC municipal entre 2002 e 2007 :

$$
\operatorname{TPIBPC}_{m, i f}=\left(\frac{P I B P C_{m, f}}{P I B P C_{m, i}}\right)^{1 / n}-1
$$

em que: TPIBPC $C_{m, j}$ é a taxa anual de crescimento do Produto interno bruto entre 2002 e 2007; PIBPC $_{m, f}$, o produto interno bruto municipal de 2007; PIBPC $_{m, \text { i, o }}$ produto interno bruto municipal de 2002 e $n$, o intervalo em anos.

Índice de terciarização (ITc) 
Em localidades cuja estrutura dos rendimentos dos setores econômicos é por demais esdrúxula, como é o caso das chamadas cidades dormitórios e das áreas de baixíssima densidade demográfica, podem ocorrer sérias distorções no cálculo do IT, para evitá-las, empregar-se-á um fator de ponderação, capaz de expressar simultaneamente um alto nível de atividade terciária, bem como um grande volume de atividades diretamente produtivas, ou seja, um conversor logarítmico de escala, que atribui ao maior PIB, denominado PIB referencial, o fator 0,95; O ITc pode ser representado de acordo com a seguinte expressão:

$$
I T c_{m}=\frac{\mathrm{a} \cdot \mathrm{v}_{\mathrm{m}, \mathrm{s}}}{\left(\mathrm{PIB}_{\mathrm{m}}\right)}\left(1-\mathrm{e}^{-\left(\frac{-\ln (0.05)}{P I B_{r e f}} P I B_{m}\right)}\right)
$$

em que: a.v.m,s é o valor adicionado do setor de serviços no município $\mathrm{m}, \mathrm{PIB}_{\mathrm{m}}$ é o produto interno bruto municipal e $\mathrm{PIB}_{r e}$. é o produto interno bruto municipal de referência que, neste caso, foi o maior PIB municipal da UF a que pertence o município.

\section{Índice de industrialização (IDc):}

O IDc pode ser representado, analogamente ao ITc, de acordo com a seguinte expressão:

$$
I D c_{m}=\frac{\mathrm{a} \cdot \mathrm{v} \cdot \cdot_{\mathrm{m}, \mathrm{i}}}{\left(\mathrm{PIB}_{\mathrm{m}}\right)}\left(1-\mathrm{e}^{-\left(\frac{-\ln (0.05)}{P I B_{r e f}} P I B_{m}\right)}\right)
$$

em que: a.v.m,i é o valor adicionado do segundo setor no município $\mathrm{m}, \mathrm{PIB}_{\mathrm{m}}$ é o produto interno bruto municipal e $\mathrm{PIB}_{r e f}$ é o produto interno bruto municipal de referencia que, neste caso, foi o maior PIB municipal da UF a que pertence o município.

\section{Índice da dinâmica migratória municipal (IDM):}

O IDM pode ser calculado de acordo com a seguinte expressão:

$$
I D M_{m}=\frac{\sum \mathrm{P}_{\mathrm{i}} \mathrm{Ta}_{\mathrm{m}} \mathrm{Sm}_{\mathrm{m}} \mathrm{Te}_{\mathrm{im}}+\sum \mathrm{P}_{\mathrm{m}} \mathrm{Ta}_{\mathrm{i}} \mathrm{Sm}_{\mathrm{i}} \mathrm{Te}_{\mathrm{mi}}}{P I B_{m}}
$$

na qual: $P_{m}$ representa a população residente no município $m, P_{i}$ representa a população residente no município i $\mathrm{Te}_{\mathrm{mi}}$ representa a taxa de emigração do município $\mathrm{m}$ para o município $\mathrm{i}$, $\mathrm{Te}_{\mathrm{im}}$ representa a taxa de emigração do município i para o município $\mathrm{m}$, $\mathrm{Ta}_{\mathrm{m}}$ representa a taxa de atividade no munićpio $\mathrm{m}, \mathrm{Ta}_{\mathrm{i}}$ representa a taxa de atividade no município $\mathrm{i}, \mathrm{Sm}_{\mathrm{m}}$ e $\mathrm{Sm}_{\mathrm{i}}$ representam, respectivamente, o salário médio mensal auferido em município.

\section{Índice de centralidade municipal (ICM):}

O ICM pode ser calculado de acordo com a seguinte expressão:

$$
I C M_{m}=\sum \mathrm{L}_{\mathrm{im}}
$$


em que: $\mathrm{L}_{\text {im }}$ é o município i que envia uma parcela de sua população para estudar ou trabalhar no município $\mathrm{m}$.

\section{Índice de exposição ao comércio exterior (IECE):}

Esse indicador foi obtido pela razão entre o valor total das exportações internacionais do município e o PIB municipal.

$$
\mathrm{IECE}_{\mathrm{m}}=\frac{\mathrm{VCE}_{\mathrm{m}}}{\mathrm{PIB}_{\mathrm{m}}}
$$

em que: $\mathrm{VCE}_{\mathrm{m}}$ é o valor total das exportações internacionais do município $\mathrm{m}$ e $\mathrm{PIB}_{\mathrm{m}}$, o produto interno bruto municipal.

\section{Índice de capacitação tecnológica regional (ICTR)}

O ICTR tenta captar a qualificação tecnológica da mão-de-obra em cada localidade; para tanto, procurou-se mensurar e hierarquizar as diversas "características tecnológicas" presentes na descrição das ocupações efetuadas pelo Ministério do Trabalho e Emprego. Essas categorias foram: nível de escolaridade, ações tecnológicas e recursos de trabalho tecnológicos. A partir dessa metodologia, foi possível classificar as ocupações em três níveis tecnológicos: alto, médio e baixo; para efeito do cálculo do ICTR, utilizou-se apenas o nível mais alto. A partir desses indicadores, foi calculado o Índice de Capacitação Tecnológica Regional (ICTR) normalizando-se todos os indicadores listados acima, de modo que a localidade com melhor posição apresenta o valor máximo 1 .

\section{Análise Discriminante}

Análise Discriminante é um termo abrangente, que se refere a diversas técnicas estatísticas relacionadas. É uma técnica estatística para diferenciar grupos, utilizando uma derivação de regra para designar de forma ótima um novo objeto às classes existentes. Vale dizer, conhecidas as características de um novo indivíduo, pode-se prever a que grupo pertence sendo a existência de grupos conhecida a priori. No nosso caso, a existência de uma variável canônica por excelência, que nos serve de parâmetro para reclassificações, ou seja, a hierarquia da REGIC, permite identificar os indivíduos (municípios e áreas de concentração da população) que possuem probabilidade de ser classificados em níveis superiores e inferiores da hierarquia urbana e é este diagnóstico que interessa aqui.

As Funções Canônicas Discriminantes são funções lineares que combinam as variáveis discriminantes, sendo uma técnica para redução de dimensionalidade, relacionada à ACP e correlação canônica. Formalmente pode ser identificada por:

$$
\mathrm{f}_{\mathrm{km}}=\mathrm{u}_{0}+\mathrm{u}_{1} \mathrm{X}_{1 \mathrm{~km}}+\mathrm{u}_{2} \mathrm{X}_{2 \mathrm{~km}}+\ldots+\mathrm{u}_{\mathrm{p}} \mathrm{X}_{\mathrm{pkm}}
$$


na qual: $f_{k m}$ é o valor (score) da função discriminante canônica para o caso $m$ no grupo k; $\mathrm{X}_{\mathrm{ikm}}$ é o valor da variável discriminante $\mathrm{Xi}$ para caso m no grupo $\mathrm{k}$; $\mathrm{u}_{\mathrm{i}}$ são os coeficientes que produzem as características desejadas na função.

Utiliza-se a matriz das médias de cada grupo e das somas dos quadrados (intra e intergrupos) para comparar as diferenças entre eles. Feito isto, utiliza-se a matriz de correlação ou de covariância para avaliar o quanto cada variável independente pode discriminar entre os grupos, devendo-se analisar os coeficientes estandardizados para evitar problemas de unidades diferentes entre as variáveis independentes.

\section{3 - RESULTADOS}

A Tabela 1 trás os resultados da variação da hierarquia urbana - classificada em ternos de Metropole, Capital Regional, Centro Sub-regional, Centro de Zona e Centro Local - no Brasil e nas 121 sub-regiões de desenvolvimento elencadas neste estudo. A análise da tabela revela que, dada a tendência geral da economia brasileira, o país terá uma variação negativa de seus centros locais da ordem de 15\% aproximadamente, passando dos atuais 4513 para 3848 em 2020; o inverso ocorre com os Centros de Zona e os Centros Sub-regionais, que elevaram seu contingente em, respectivamente, $86 \%$ e $25 \%$; já os centros urbanos superiores, capitais regionais e metrópoles, praticamente não apresentaram variação significativa. No tocante ao produto interno bruto no período, é esperada uma variação de aproximadamente 115\%, segundo o modelo EGC. Por fim, a Figura 1, ilustra a distribuição espacial da variação dos níveis de centralidade urbana dos municípios brasileiros ao longo do território nacional. 
Tabela 1. Brasil: 2007-2020. Variação da Hierarquia Urbana e do Produto Interno Bruto nas Subregiões de Desenvolvimento.

\begin{tabular}{|c|c|c|c|c|c|c|c|c|c|c|c|c|c|}
\hline \multirow[b]{2}{*}{ Sub-regiōes } & \multicolumn{6}{|c|}{ Regiōes de Influência das Cidades (REGIC) - 2007} & \multicolumn{6}{|c|}{ Simulação: 2020} & \multirow[b]{2}{*}{$\begin{array}{c}\text { Variação } \\
\text { do PIB } \\
(\%)\end{array}$} \\
\hline & Metropole & $\begin{array}{l}\text { Capital } \\
\text { Regional }\end{array}$ & $\begin{array}{l}\text { Centro Sub- } \\
\text { regional }\end{array}$ & $\begin{array}{l}\text { Centro de } \\
\text { Zona }\end{array}$ & Centro Local & $\begin{array}{c}\text { PIB (milhöes } \\
\text { de Reais) }\end{array}$ & Metropole & $\begin{array}{c}\text { Capital } \\
\text { Regional }\end{array}$ & $\begin{array}{c}\text { Centro } \\
\text { Sub- } \\
\text { regional }\end{array}$ & $\begin{array}{c}\text { Centro de } \\
\text { Zona }\end{array}$ & $\begin{array}{l}\text { Centro } \\
\text { Local }\end{array}$ & $\begin{array}{c}\text { PIB } \\
\text { (milhões } \\
\text { de Reais) } \\
\end{array}$ & \\
\hline Abaetetuba & & & \multicolumn{2}{|l|}{2} & 14 & 2323 & & & 2 & 4 & 10 & 4259 & 83,35 \\
\hline Alta Floresta & & & & 4 & 10 & 2029 & & & & 9 & 5 & 3789 & 86,70 \\
\hline Altamira & & & 1 & & 7 & 1166 & & & 1 & 4 & 3 & 2161 & 85,38 \\
\hline Alto Solimões & & & & 1 & 6 & 585 & & & & 1 & 6 & 1322 & 126,23 \\
\hline Aracaju & & 1 & 3 & 5 & 33 & 12810 & & 1 & 4 & 13 & 24 & 29343 & 129,06 \\
\hline Araçatuba & & 1 & 1 & 6 & 37 & 10305 & & & 2 & 5 & 38 & 18327 & 77,84 \\
\hline Araguaina & & 1 & & 3 & 38 & 3045 & & 1 & 1 & 22 & 18 & 7768 & 155,12 \\
\hline Arapiraca & & 1 & & 2 & 25 & 3003 & & 1 & & 2 & 25 & 5489 & 82,83 \\
\hline Arcoverde & & & 3 & 3 & 18 & 1938 & & & 2 & 4 & 18 & 3965 & 104,58 \\
\hline Bagé-Uruguaiana & & & 2 & 7 & 18 & 9884 & & & 2 & 6 & 19 & 17285 & 74,88 \\
\hline Balsas & & & 1 & 2 & 10 & 1228 & & & 1 & 1 & 11 & 2239 & 82,34 \\
\hline Barbacena & & & 2 & & 25 & 3322 & & & 2 & 1 & 24 & 6475 & 94,89 \\
\hline Barra do Garças & & & 1 & 1 & 16 & 2540 & & & 1 & 7 & 10 & 4750 & 87,02 \\
\hline Barreiras & & 1 & & 3 & 20 & 5384 & & & 1 & 2 & 21 & 8098 & 50,42 \\
\hline Bauru & & 1 & 4 & 5 & 56 & 21968 & & 1 & 3 & 11 & 51 & 39340 & 79,08 \\
\hline Belém & 1 & 1 & 5 & 3 & 38 & 25497 & & 2 & 6 & 16 & 24 & 64541 & 153,13 \\
\hline Belo Horizonte & 1 & 3 & 6 & 32 & 170 & \begin{tabular}{|l|}
114021 \\
\end{tabular} & 1 & 2 & 9 & 39 & 161 & 247580 & 117,14 \\
\hline Blumenau & & 1 & 4 & 6 & 43 & 28322 & & 2 & 6 & 18 & 28 & 57897 & 104,42 \\
\hline Boa Vista & & 1 & & & 5 & 3400 & & 1 & & 3 & 2 & 8044 & 136,57 \\
\hline Bom Jesus da Lapa & & & 1 & & 5 & 614 & & & & 2 & 4 & 1133 & 84,55 \\
\hline Brasilia & 1 & & 1 & 16 & 42 & 110414 & 1 & & 2 & 21 & 36 & 210051 & 90,24 \\
\hline Cabeça de Cachorro & & & & & 3 & 277 & & & & 1 & 2 & 668 & 141,47 \\
\hline Campina Grande & & 1 & & 4 & 67 & 5380 & & 1 & & 11 & 60 & 10666 & 98,26 \\
\hline Campinas & & 2 & 15 & 15 & 62 & 120142 & 1 & & 16 & 31 & 46 & 234909 & 95,53 \\
\hline Campo Grande & & 1 & & 10 & 28 & 17018 & & 1 & 1 & 18 & 19 & 31045 & 82,43 \\
\hline Campos dos Goytaca & & 1 & 1 & 1 & 9 & 24499 & & & 2 & & 10 & 51029 & 108,29 \\
\hline Caruaru & & 1 & 1 & 3 & 56 & 8156 & & 1 & 3 & 8 & 49 & 16433 & 101,48 \\
\hline Cascavel & & 1 & 3 & 8 & 76 & 24380 & & 1 & 3 & 16 & 68 & 46445 & 90,50 \\
\hline Caxias & & & 2 & 3 & 22 & 2933 & & & 1 & 8 & 18 & 5743 & 95,82 \\
\hline Caxias do Sul & & 1 & 2 & 8 & 42 & 20869 & & 1 & 2 & 13 & 37 & 43447 & 108,19 \\
\hline Ceará Meridional & & 1 & 2 & 5 & 45 & 5382 & & & 2 & 17 & 34 & 11377 & 111,38 \\
\hline Chapecó & & 1 & 6 & 12 & 99 & 20079 & & 1 & 5 & 17 & 95 & 35811 & 78,35 \\
\hline Cruzeiro do Sul & & & 1 & 2 & 10 & 1441 & & & 1 & 3 & 9 & 3132 & 117,36 \\
\hline Cuiabá & & 2 & & 6 & 20 & 16864 & & $2 \mid$ & 4 & 12 & 10 & 41737 & 147,50 \\
\hline Curitiba & 1 & & 7 & 20 & 72 & 84168 & 1 & & 5 & 32 & 62 & 194427 & 131,00 \\
\hline Divinópolis & & 1 & & 7 & 32 & 9201 & & & 2 & 10 & 28 & 17838 & 93,88 \\
\hline Dourados & & 1 & & 6 & 29 & 8802 & & 1 & 2 & 12 & 21 & 14300 & 62,47 \\
\hline Feira de Santana & & 1 & 4 & 9 & 91 & 13901 & & 1 & 2 & 20 & 82 & 28732 & 106,69 \\
\hline Floriano-Uruçui & & & 2 & 9 & 51 & 2090 & & & 1 & 11 & 50 & 3845 & 84,01 \\
\hline Florianópolis & & 2 & 2 & 3 & 14 & 14174 & & $2 \mid$ & 3 & 3 & 13 & 29930 & 111,17 \\
\hline Fortaleza & 1 & & 2 & 7 & 37 & 35724 & 1 & & 1 & 14 & 31 & 91737 & 156,80 \\
\hline Goiânia & 1 & & 4 & 26 & 130 & 45806 & 1 & 1 & 6 & 49 & 104 & 111851 & 144,18 \\
\hline Governador Valadares & & 1 & & 6 & 47 & 4800 & & 1 & & 7 & 46 & 9223 & 92,16 \\
\hline Guarapuava & & & 2 & 6 & 31 & 8512 & & & 3 & 4 & 32 & 15822 & 85,88 \\
\hline Ilhéus-Porto Seguro & & 1 & 3 & 6 & 50 & 11173 & & & 5 & 24 & 31 & 22624 & 102,49 \\
\hline Imperatriz & & 1 & & 5 & 40 & 6371 & & 1 & 1 & 14 & 30 & 13409 & 110,49 \\
\hline Ipatinga & & 1 & 2 & 3 & 27 & $\begin{array}{l}10727 \\
\end{array}$ & & & 3 & 6 & 24 & 24344 & 126,93 \\
\hline Irecê & & & 1 & 6 & 48 & 3471 & & & & 9 & 46 & 6496 & 87,13 \\
\hline Itaituba & & & 1 & & 5 & 960 & & & 1 & 2 & 3 & 1944 & 102,45 \\
\hline Itapetininga & & & 3 & 2 & 34 & 7842 & & & 1 & 6 & 32 & 12745 & 62,53 \\
\hline Jaguaribe & & & & 4 & 13 & 1989 & & & & 6 & 11 & 4475 & 125,01 \\
\hline Ji-Paraná & & & 2 & 3 & 19 & 5610 & & 1 & 3 & 11 & 9 & 13014 & 131,99 \\
\hline João Pessoa & & 2 & 3 & 4 & 59 & 13607 & & 2 & 3 & 12 & 51 & 27734 & 103,83 \\
\hline Joinville & & 2 & 1 & 6 & 17 & 25602 & & 2 & 3 & 10 & 11 & 52984 & 106,95 \\
\hline Juiz de Fora & & 1 & 3 & 10 & 75 & 15721 & & 1 & 1 & 20 & 67 & 32343 & 105,73 \\
\hline Lábrea & & & & 1 & 3 & 449 & & & & 1 & 3 & 1055 & 135,15 \\
\hline Lages & & & 1 & 2 & 27 & 5027 & & & 1 & 6 & 23 & 9291 & 84,83 \\
\hline Londrina & & 1 & 1 & 9 & 41 & 18067 & & 1 & 3 & 8 & 40 & 36716 & 103,21 \\
\hline Macapá & & 2 & & & 9 & 5557 & & $2 \mid$ & 1 & 4 & 4 & 10827 & 94,85 \\
\hline & & & & & & & & & & & & & continua \\
\hline
\end{tabular}




\begin{tabular}{|c|c|c|c|c|c|c|c|c|c|c|c|c|c|}
\hline \multirow[b]{2}{*}{ Sub-regiōes } & \multicolumn{6}{|c|}{ Regiōes de Influência das Cidades (REGIC) - 2007} & \multicolumn{6}{|c|}{ Simulação: 2020} & \multirow[b]{2}{*}{$\begin{array}{c}\text { Variação } \\
\text { do PIB } \\
(\%)\end{array}$} \\
\hline & Metropole & $\begin{array}{c}\text { Capital } \\
\text { Regional }\end{array}$ & $\begin{array}{l}\text { Centro Sub- } \\
\text { regional }\end{array}$ & $\begin{array}{l}\text { Centro de } \\
\text { Zona }\end{array}$ & Centro Local & $\begin{array}{c}\text { PIB (milhões } \\
\text { de Reais) }\end{array}$ & Metropole & $\begin{array}{c}\text { Capital } \\
\text { Regional }\end{array}$ & $\begin{array}{c}\text { Centro } \\
\text { Sub- } \\
\text { regional }\end{array}$ & $\begin{array}{c}\text { Centro de } \\
\text { Zona }\end{array}$ & $\begin{array}{l}\text { Centro } \\
\text { Local }\end{array}$ & \begin{tabular}{|c|} 
PIB \\
(milhões \\
de Reais) \\
\end{tabular} & \\
\hline Maceió & & 1 & & 5 & 39 & 13309 & & 1 & & 10 & 34 & \begin{tabular}{|l|}
25269 \\
\end{tabular} & 89,87 \\
\hline Manaus & 1 & & & & 15 & 37220 & & 1 & & 2 & 13 & 115337 & 209,88 \\
\hline Manicoré & & & 1 & 1 & 14 & 2141 & & & & 3 & 13 & 4766 & 122,64 \\
\hline Marabá & & 1 & 2 & 1 & 20 & 12189 & & 1 & 6 & 12 & 5 & 30801 & 152,69 \\
\hline Marajó & & & 1 & & 11 & 819 & & & & 1 & 11 & 1460 & 78,34 \\
\hline Marilia & & 1 & 1 & 4 & 39 & 11382 & & 1 & 3 & 6 & 35 & 19908 & 74,90 \\
\hline Maringá & & 1 & 4 & 6 & 98 & 18783 & & 1 & 3 & 20 & 85 & 36730 & 95,55 \\
\hline Montes Claros & & 1 & 1 & 9 & 78 & 9636 & & 1 & 1 & 11 & 76 & 18558 & 92,58 \\
\hline Mossoró & & 1 & 1 & 1 & 31 & 6086 & & 1 & 3 & 9 & 21 & 16581 & 172,45 \\
\hline Natal & & 2 & 1 & 10 & 55 & 14060 & & 2 & 1 & 16 & 49 & 31839 & 126,45 \\
\hline Norte Capixaba & & & 3 & 4 & 25 & 12318 & & & 4 & 11 & 17 & 29446 & 139,05 \\
\hline Oiapoque & & & & & 5 & 465 & & & & 2 & 3 & 934 & 100,65 \\
\hline Ouricuri-Araripina & & & 1 & 1 & 8 & 930 & & & 1 & 4 & 5 & 1937 & 108,25 \\
\hline Palmas & & 1 & 1 & 11 & 84 & 8049 & & 2 & 6 & 37 & 52 & 19684 & 144,55 \\
\hline Pantanal & & & 1 & 3 & 15 & 4350 & & & 2 & 7 & 10 & 7905 & 81,72 \\
\hline Passo Fundo & & 1 & 4 & 18 & 137 & 20003 & & 1 & 3 & 13 & 143 & 36928 & 84,61 \\
\hline Patos & & & 4 & 8 & 71 & 3215 & & & 3 & 13 & 67 & 6239 & 94,06 \\
\hline Paulo Afonso & & & 2 & 4 & 35 & 5389 & & & 2 & 8 & 31 & 10920 & 102,61 \\
\hline Pelotas & & 1 & 1 & 1 & 14 & 10194 & & 1 & 1 & & 15 & 20130 & 97,46 \\
\hline $\begin{array}{l}\text { Petrolina-Juazeiro } \\
\end{array}$ & & 1 & 2 & 1 & 28 & 7147 & & 1 & 2 & 9 & 20 & 13975 & 95,55 \\
\hline Picos & & & 1 & 10 & 86 & 2475 & & & 1 & 15 & 81 & 4778 & 93,03 \\
\hline Ponta Grossa & & 1 & & 2 & 8 & 7671 & & 1 & & 3 & 7 & 15058 & 96,29 \\
\hline Porto Alegre & 1 & 3 & 10 & 31 & 107 & 100141 & 1 & 3 & 12 & 43 & 93 & 221532 & 121,22 \\
\hline Porto Velho & & 1 & 1 & 1 & 14 & 7346 & & 1 & 2 & 11 & 3 & 18001 & 145,03 \\
\hline Pouso Alegre & & 1 & 1 & 3 & 43 & 7623 & & & 3 & 4 & 41 & 14867 & 95,02 \\
\hline Presidente Prudente & & 1 & & 7 & 46 & 9863 & & 1 & & 12 & 41 & 17756 & 80,02 \\
\hline Quixadá & & & 2 & 2 & 25 & 2632 & & & & 8 & 21 & 5532 & 110,19 \\
\hline Recife & 1 & 1 & 8 & 11 & 47 & 47172 & 1 & 1 & 12 & 18 & 36 & 113965 & 141,59 \\
\hline Ribeirão Preto & & 2 & 3 & 12 & 89 & 53666 & & 2 & 7 & 26 & 71 & 98233 & 83,05 \\
\hline Rio Branco & & 1 & & 2 & 13 & 4842 & & 1 & & 7 & 8 & 10573 & 118,39 \\
\hline Rio de Janeiro & 1 & 1 & 10 & 15 & 36 & 252685 & 1 & 2 & 10 & 25 & 25 & 639411 & 153,05 \\
\hline Rio Verde & & & 1 & 3 & 23 & 8486 & & 1 & 1 & 6 & 19 & 16266 & 91,68 \\
\hline Rondonópolis & & & 1 & 3 & 22 & 9710 & & 1 & 1 & 12 & 12 & 20996 & 116,23 \\
\hline Roraima Setentrional & & & & & 4 & 326 & & & & \begin{tabular}{l|l}
3 \\
\end{tabular} & 1 & 852 & 161,57 \\
\hline Rorainópolis & & & & & 5 & 443 & & & & 5 & & 1165 & 163,22 \\
\hline Salvador & 1 & 1 & 7 & 6 & 38 & 59591 & 1 & 1 & 6 & 22 & 23 & 155177 & 160,41 \\
\hline Santa Luzia & & & 5 & 10 & 65 & 6107 & & & 2 & 15 & 63 & 11772 & 92,77 \\
\hline Santa Maria & & 2 & 2 & 7 & 76 & 15523 & & 1 & 3 & 8 & 75 & 27518 & 77,27 \\
\hline Santarém & & 1 & & 2 & 11 & 3692 & & & 3 & 1 & 10 & 8238 & 123,15 \\
\hline São Felix do Araguaia & & & & 3 & 11 & 948 & & & & 6 & 8 & 1673 & 76,61 \\
\hline São Félix do Xingu & & & 1 & 3 & 11 & 2862 & & & 5 & 3 & 7 & 5096 & 78,07 \\
\hline São José do Rio Preta & & 1 & 2 & 8 & 92 & 23634 & & 1 & 6 & 9 & 87 & 41656 & 76,26 \\
\hline São José dos Campo & & 1 & 4 & 5 & 20 & 40245 & & & 4 & 9 & 17 & 83184 & 106,70 \\
\hline São Luís & & 1 & & 10 & 40 & 14967 & & 1 & & 9 & 41 & 35175 & 135,01 \\
\hline São Paulo & 1 & 5 & 15 & 29 & 20 & 581985 & 1 & 6 & 15 & 33 & 15 & \begin{tabular}{|l|}
1174463 \\
\end{tabular} & 101,80 \\
\hline Seridó & & & 2 & 1 & 21 & 1323 & & & 2 & 4 & 18 & 2855 & 115,72 \\
\hline Sertão Alagoano & & & 1 & 4 & 24 & 1482 & & & & 5 & 24 & 2881 & 94,41 \\
\hline Sertão Sergipano & & & 1 & 4 & 28 & 4085 & & & 2 & 6 & 25 & 7550 & 84,81 \\
\hline Sinop & & & 1 & 4 & 19 & 7578 & & 1 & 2 & 13 & 8 & 14798 & 95,27 \\
\hline Sobral & & 1 & 1 & 6 & 44 & 5674 & & & 2 & 13 & 37 & 11821 & 108,32 \\
\hline Sorocaba & & 1 & 1 & 12 & 15 & 31951 & & & 3 & 16 & 10 & 63854 & 99,85 \\
\hline Sudoeste Potiguar & & & 1 & 5 & 35 & 1456 & & & & 7 & 34 & 3428 & 135,39 \\
\hline Tefé & & & 1 & 1 & 7 & 831 & & & & 2 & 7 & 1747 & 110,30 \\
\hline Teófilo Otoni & & 1 & & 10 & 63 & 4867 & & & 2 & 10 & 62 & 9135 & 87,71 \\
\hline Teresina & & 1 & 1 & 9 & 39 & 8501 & & 1 & & 8 & 41 & 18947 & 122,88 \\
\hline Tubarão & & 1 & 2 & 3 & 38 & 11420 & & 1 & 2 & 17 & 24 & 22278 & 95,08 \\
\hline Uberlândia & & 2 & 2 & 10 & 63 & 40904 & & \begin{tabular}{l|l}
3 & \\
\end{tabular} & 7 & \begin{tabular}{l|l}
6 \\
\end{tabular} & 61 & 78773 & 92,58 \\
\hline Varginha & & 1 & 2 & 4 & 30 & 9800 & & 1 & 4 & 4 & 28 & 18504 & 88,81 \\
\hline $\begin{array}{l}\text { Vilhena } \\
\end{array}$ & & & 1 & 2 & 16 & 3387 & & & 1 & 8 & 10 & 7541 & 122,66 \\
\hline Vitória & & 2 & 2 & 6 & 36 & 48022 & & 1 & 3 & 21 & 21 & 141332 & 194,31 \\
\hline Vitória da Conquista & & 1 & 2 & 6 & 54 & 7041 & & 1 & & 16 & 46 & 14306 & 103,18 \\
\hline Volta Redonda & & 1 & 3 & 11 & 31 & 20135 & & & 4 & 9 & 33 & 45009 & 123,54 \\
\hline Brasil & 12 & 89 & 243 & 707 & 4513 & 2661345 & 11 & 82 & 304 & 1319 & 3848 & \begin{tabular}{|l|}
5721050 \\
\end{tabular} & 114,97 \\
\hline
\end{tabular}

Fonte: IBGE. Censos Demográficos de 2000 e 2010; Produto Interno Bruto Municipal de 2007; Região de Influência das Cidades de 2007. 


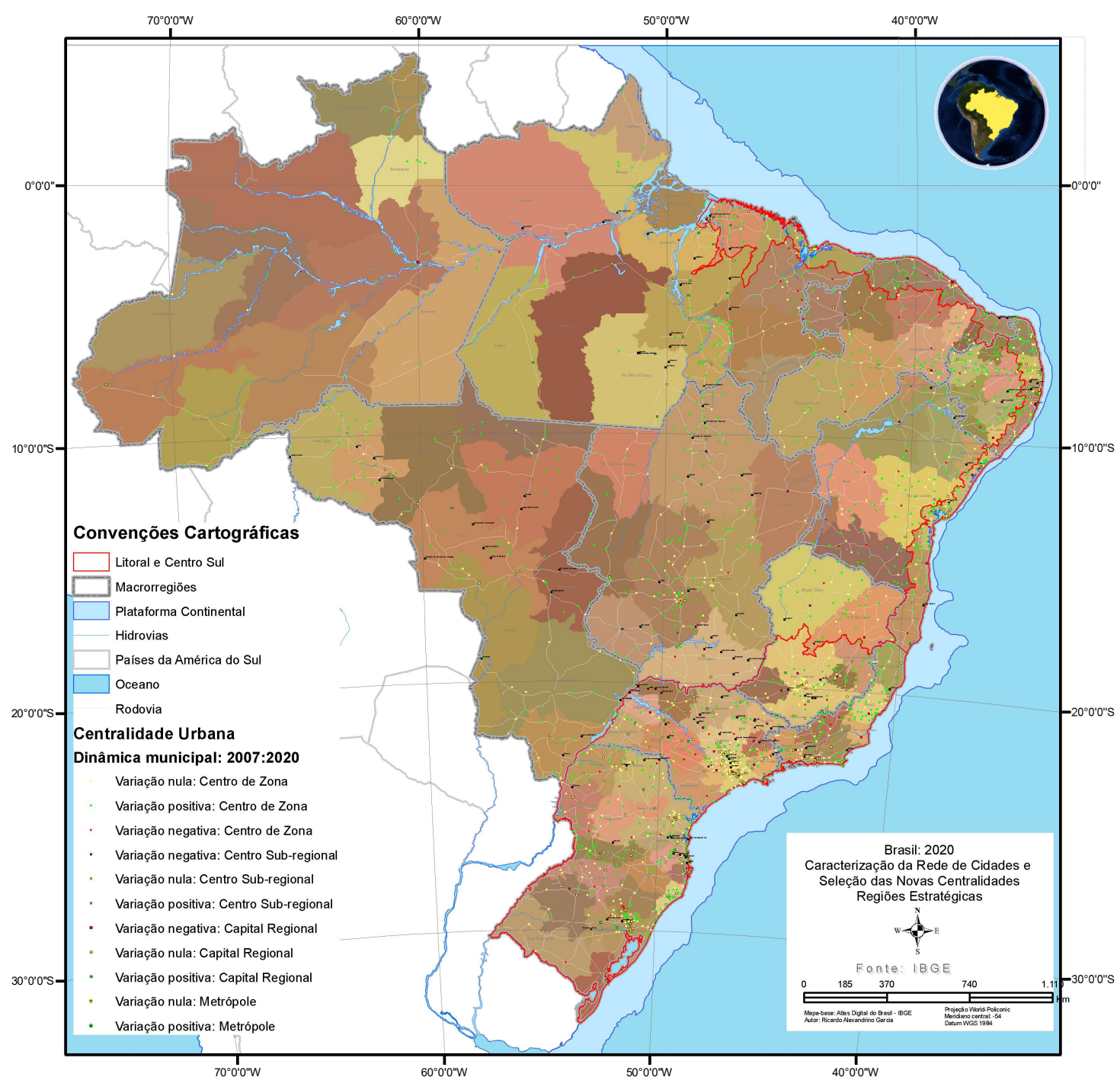

Figura 1. Brasil 2020: Caracterização da Rede de Cidades e Seleção de Novas Centralidades segundo Regiões Estratégicas de Desenvolvimento. Fonte: IBGE. Censos Demográficos de 2000 e 2010; Produto Interno Bruto Municipal de 2007; Região de Influência das Cidades de 2007.

\section{4 - CONSIDERAÇÕES FINAIS}

A simulação do sistema urbano e, principalmente, a análise de suas diferenças para o identificado pelo REGIC/2007, possibilitaram a identificação prospectiva das tendências da rede urbana brasileira. Neste sentido, a caracterização da rede de cidades operacionalizada através da regionalização é apenas um primeiro passo metodológico. Para a seleção final das novas centralidades seria necessário construir um consenso dos atores atuantes no território balizando as escolhas dos novos polos em escala macro e meso espacial. Esses atores são constituídos por formuladores de políticas do setor público, formadores de opinião representantes da sociedade civil, como ONGs e sindicatos trabalhistas, estudiosos especialistas e empresários investidores potenciais no território

Esta é a forma de progressão do chamado planejamento tradicional para o planejamento situacional, que trás os atores para o palco principal do planejamento territorial. No 
entanto, as limitações de tempo e recursos impedem tal empreitada. Uma forma eficaz de mitigação dessa restrição é a metodologia de cenários. Este método é uma representação que também situa o planejador no contexto político-institucional dos atores no território. Por esse procedimento, os indicadores de caracterização do território podem ser analisados por uma representação dos atores, através de alguns representantes do governo federal, dos governos estaduais e especialistas no território.

A partir dessa abordagem dos indicadores hipóteses podem ser elaboradas e algumas regras são previamente estabelecidas nesse jogo territorial. No caso em questão, a premissa geral buscou uma configuração territorial menos desigual e mais policêntrica sob a restrição de desenvolvimento sustentável de baixo carbono. A segunda premissa buscou uma configuração territorial mais igualitária e que fortaleça a coesão territorial do país e regiões. A terceira foi combinar a maior coesão territorial interna do país com os objetivos da integração sul-americana, especialmente em sua dimensão da integração física, da livre mobilidade de pessoas e do compartilhamento da exploração de recursos naturais comuns. Partindo dessas premissas as escolhas puderam ser feitas e a seleção das centralidades operacionalizada.

\section{REFERÊNCIAS BIBLIOGRÁFICAS}

BAROUCHE, J.M. \& SAPORTA, G. Análise de dados. Rio de Janeiro: Zahar, 1982.

BAUDELLE, G.; PEYRONY, J. Striving for equity: polycentric development policies in France. Built Environment, v. 31, n. 2, p. 103-111, 2005.

BRASIL. Estudo da Dimensão Territorial para o Planejamento: Volume III. In: Estudo para subsidiar a abordagem da dimensão territorial do desenvolvimento nacional no Plano Plurianual PPA 2008-2011 e no planejamento governamental de longo prazo. Regiões de Referência / Ministério do Planejamento, Orçamento e Gestão. Secretaria de Planejamento e Investimentos Estratégicos. Brasília: MP, 2008.

CASTELLS, M. The rise of the network society. The informational age: economy, society and culture. Cambridge: Basil Blackwell, 1996.

CASTRO, A. B. de. A Herança Regional no Desenvolvimento Brasileiro. In: CASTRO, A. B. de 1980. Sete Ensaios sobre a Economia Brasileira. Rio de Janeiro: Forense Universitária, $3^{\text {a }}$ Ed., p. 09-83, 1970.

CENTRO DE GESTÃO E ESTUDOS ESTRATÉGICOS - CGEE. Caracteriqação da Rede de Cidades e Seleção das Novas Centralidades. Relatório de Pesquisa. Brasília: CGEE, 2011. 43p. 
CORREA, R. L. Região e organiz̨ação espacial. São Paulo: Ática, 1987.

CUNHA, A. M.; SIMÕES, R.; PAULA, J. A. História Econômica e Regionalização: contribuição a um desafio metodológico. Estudos Econômicos. Instituto de Pesquisas Econômicas, v. 38, p. 02-22, 2008.

DINIZ, C. C. A busca de um projeto de nação: o papel do território e das políticas regional e urbana. Revista da Anpec, v.7, n.4, p.1-18, 2006.

DINIZ, C.C. A nova geografia econômica do Brasil: condicionantes e implicações. In: VELLOSO, J.P.R. (org). Brasil século XXI. Rio de Janeiro: José Olympio, 2000.

DINIZ, C. C. Desenvolvimento poligonal no Brasil: nem desconcentração, nem contínua polarização. Nova Economia, v. 3, n. 1, set. p. 35-64, 1993.

GARCIA, R., LEMOS, M. A Migração como Variável Endógena: Caracterização do Processo de Transformação das Regiões de Influência dos Pólos Econômicos Brasileiros. Revista Economia, v.10, n.2, p.253-275, 2009.

ISARD, W. Methods of regional analysis. Cambridge: MIT Press, 1975.

KAGEYAMA, A. \& LEONE, E.T. Uma tipologia de municipios paulistas com base em indicadores sociodemográficos. Campinas: UNICAMP/IE, 1999. (Textos para discussão; nº 66)

KLECKA, W. R. 1980. Discriminant Analysis. Sage University Paper series on Quantitative Applications in the Social Sciences. Beverly Hills, CA: Sage.

LEMOS, Maurício Borges. Duas técnicas de análise regional elaboradas a partir de categorias espaciais: a regionalização e o método estrutural-diferencial. Belo Horizonte, DCE/ FACE/UFMG, 1991. (Tese de Professor Titular).

LEMOS, M., DINIZ, C. C., GUERRA, L., MORO, S. A Nova Configuração Regional Brasileira e sua Geografia Econômica. Estudos Econômicos, v. 33, n. 4, p. 665-700, 2003. 
MAGNAGO, Angélica Alves. A divisão regional brasileira - uma revisão bibliográfica. Revista Brasileira de Geografia, Rio de Janeiro, v. 57, n. 4, p. 65-92, out./dez. 1995.

MAINLY, B.F.J. Multivariate statistical methods: a primer. London: Chapman and Hall, 1986.

MC'LACHLAN, G. J. 2004. Discriminant Analysis and Statistical Pattern Recognition. John Wiley \& Sons, Inc., Hoboken, New Jersey.

PACHECO, C.. Desconcentração econômica e fragmentação da economia nacional. Economia e Sociedade, v. 6, p. 113-140, 1996.

REGIC. Região de influência das cidades. Rio de Janeiro, IBGE, 2008. 